\title{
Fruiting bodies of selected edible mushrooms as a potential source of lovastatin
}

\author{
Katarzyna Kała ${ }^{1} \cdot$ Agata Kryczyk-Poprawa $^{2}$ (1) $\cdot$ Aleksandra Rzewińska ${ }^{1} \cdot$ Bożena Muszyńska ${ }^{1}$
}

Received: 25 September 2019 / Revised: 3 January 2020 / Accepted: 12 January 2020 / Published online: 25 January 2020

(c) The Author(s) 2020

\begin{abstract}
Agaricus bisporus, Cantharellus cibarius, Imleria badia, and Lentinula edodes are among the most popular species of edible mushrooms in Poland. These edible mushrooms are an important source of biologically active substances exhibiting beneficial (e.g., antioxidant, antitumor, antimicrobial, anti-inflammatory) effects on the human body. The fruiting bodies of edible mushrooms are also a valuable source of lovastatin, which belongs to a group of compounds, called statins, commonly used as cholesterol-lowering drugs. Due to the presence of lovastatin, edible mushrooms can be useful in the prevention of hypercholesterolemia. Therefore, the aim of this study was to determine the content of lovastatin in the selected species of edible mushrooms and to evaluate its release into artificial digestive juices. This study was the first to determine the release of lovastatin into digestive juices after the extraction of materials obtained from edible mushrooms. The largest amount of lovastatin was found in the fruiting bodies of C. cibarius $(67.89 \mathrm{mg} / 100 \mathrm{~g} \mathrm{~d} . \mathrm{w}$.), and the smallest in those of L. edodes $(0.95 \mathrm{mg} / 100 \mathrm{~g}$ d.w.). The amount of lovastatin released from the extracts of the examined species into digestive juices was found to be relatively low. The highest content after incubation in artificial gastric juice was detected for the fruiting bodies of L. edodes $(0.02 \mathrm{mg} / 100 \mathrm{~g}$ d.w. $)$ and after incubation in the intestinal juice for the mycelium from the in vitro cultures of L. edodes $(0.51 \mathrm{mg} / 100 \mathrm{~g}$ d.w.). Thus, the results of the present study showed that due to its ability to accumulate lovastatin from culture medium, L. edodes mycelium can be used to obtain a product with increased hypolipidemic activity.
\end{abstract}

Keywords Lovastatin · Edible mushrooms $\cdot$ Lentinula edodes $\cdot$ Artificial digestive juice $\cdot$ Mycelial culture

\section{Introduction}

The interest in edible mushrooms has been constantly growing since the last decade due to their prohealth and therapeutic activities which are proved in many scientific experiments. Edible mushrooms are a source of valuable biologically active substances, including phenolic and indolic compounds, carotenoids, flavonoids, sesquiterpenoids, glucans, glycoproteins, triterpenoids, sterols, tocopherols, antibiotics, vitamins, and bioelements, which are present in them in significant quantities and often act synergistically. It has been proven that these substances exert

Agata Kryczyk-Poprawa

akryczyk@gmail.com

1 Department of Pharmaceutical Botany, Faculty of Pharmacy, Jagiellonian University Medical College, Kraków, Poland

2 Department of Inorganic and Analytical Chemistry, Faculty of Pharmacy, Jagiellonian University Medical College, Kraków, Poland immunostimulatory, anticancer, antibacterial, antifungal, anti-inflammatory, and antioxidant effects [1-7]. In addition to these therapeutic compounds, mushrooms contain numerous dietary components and exogenous amino acids. Moreover, the protein derived from the fruiting bodies of mushrooms is well assimilable. The high nutritional value of mushrooms is also associated with their presence of vitamins, minerals, unsaturated fatty acids, and dietary fiber [8-11]. Furthermore, the fruiting bodies of edible mushrooms are a valuable source of lovastatin which belongs to a group of compounds called statins, commonly used as cholesterol-lowering drugs [12-16]. Lovastatin is a naturally occurring compound that selectively blocks the synthesis of endogenous cholesterol. It was first isolated from Aspergillus terreus in 1978 and introduced into the medical field by Merck in 1987 [17].

Lovastatin consists of a six-part lactone ring with a hydroxyl group and a partially hydrogenated naphthalene with a hydroxyl substituent esterified with a 2-methylbutyric acid residue (Fig. 1). 


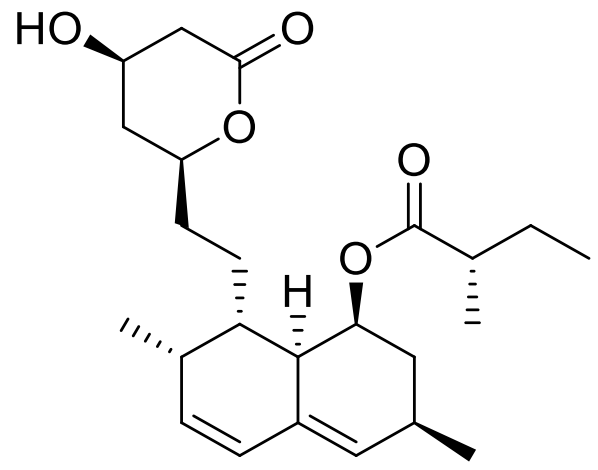

Fig. 1 Structural formula of lovastatin

Lovastatin is present in the living organisms in the form of lactone which is insoluble in water, while its active form $\beta$-hydroxy acid is water soluble. Thus, lovastatin is a precursor, which during enzymatic reactions is transformed from the form of lactone to hydroxy acid that competitively blocks 3-hydroxy 3-methyl-glutaryl-coenzyme A (HMG$\mathrm{CoA}$ ) reductase. As a result, the transformation of HMGCoA to mevalonate, which is a key stage in the synthesis of cholesterol, is blocked. This mechanism leads to a reduction in cholesterol synthesis, and hence its concentration in the liver, and increases the expression of the low-density lipoprotein receptor on the hepatocyte cell membrane, which is captured from the blood, and decreases its plasma level [17, 18]. Medicines from the statins group also show pleiotropic effects, which include protection of the vascular endothelium and stimulation of its regeneration, stabilization of the atherosclerotic plaque, influence on the clotting system, and involvement in anti-inflammatory and antioxidation processes [19]. It has now been stated that the pathophysiology of atherosclerosis is associated with the induction of inflammatory processes in the vascular wall and, therefore, statins play an important role in the prevention and treatment of this disease.

It has been shown that lovastatin can exert a multidirectional effect in various diseases such as osteoporosis, neurodegenerative diseases, rheumatoid arthritis, ischemic heart disease, and nonalcoholic fatty liver disease [20-24]. Lovastatin also exhibits promising anticancer properties by suppressing the genes involved in cell division, increasing the activity of cell cycle inhibitors and decreasing the activity of cyclins B and D1, activating caspase-3, and inhibiting antiapoptotic factors [25-27]. Lovastatin helps to prevent metastasis and, therefore, it is administered together with anticancer drugs and during radio- and chemotherapies. For instance, this compound can be used in the treatment of malignant mesothelioma since it plays a role in the prevention of cancer proliferation and angiogenesis by inhibiting the activation of vascular endothelial growth factor-2 (VEGFR-2). Thus, lovastatin, in combination with a VEGFR-2 inhibitor, can exhibit a stronger inhibitory effect on tumor growth [28]. In addition, the antifungal effect of lovastatin has been demonstrated by the growth inhibition of Rhodotorula rubra, Mucor racemosus, and Candida albicans [29].

Due to the presence of dietary substances, edible mushrooms are classified as functional foods $[9,11]$. Due to this advantage, it is possible to enrich the mycelium from in vitro cultures with appropriate precursors of active substances to obtain an increased amount of those substances. Various crop modifications are also applied; for example, in the case of Agaricus bisporus species, exposing the developing fruiting bodies to UV light leads to a high content of vitamin $\mathrm{D}_{3}$ [30]. Similarly, lovastatin content can be increased in the fruiting bodies of edible mushrooms by modifying the medium composition and optimizing the culture conditions. Mushrooms also have the ability to accumulate drugs and, hence, there is a possibility to maximize the content of desired drugs in their fruiting bodies [31-33].

The aim of this study was to determine the content of lovastatin in the lyophilized fruiting bodies of four edible mushroom species, namely A. bisporus, Cantharellus cibarius, Imleria badia, and Lentinula edodes, and in the lyophilized mycelium from the in vitro cultures of these species. Qualitative and quantitative analyses were performed using reversed-phase high-performance liquid chromatography (RP-HPLC). To determine the actual amount of lovastatin available to the human body, the release of this compound from mushroom material (fruiting bodies and mycelium from in vitro cultures) into artificial digestive juices was evaluated in a Gastroel-2014 apparatus. Thus far, there are no studies available evaluating the release of lovastatin from fruiting bodies and mycelium from the in vitro cultures of edible mushrooms into digestive juices. The present study is the first to determine the content of lovastatin in digestive juices after extraction of the fruiting bodies of edible mushrooms and mycelium from in vitro cultures. This process worked most efficiently for L. edodes and, therefore, this species was selected for further research, in which the ability of the in vitro cultures of this species to accumulate lovastatin from the culture medium was evaluated. For this purpose, the medium was enriched with lovastatin and tablets of two commercial preparations containing this compoundLiprox and Lovasterol. The results showed that bioaccumulation of lovastatin by mycelium may allow enriching the mushroom culture to obtain a product with increased anticholesterol activity. In the next stage of the study, the release of lovastatin from the lyophilized biomass of the in vitro cultures of $L$. edodes into artificial digestive juices was evaluated. 


\section{Materials and methods}

\section{Mushroom material}

The fruiting bodies of $A$. bisporus (J.E. Lange) Imbach, L. edodes (Berk.) Pegler of commercial origin, and $C$. cibarius Fr. and I. badia (Fr.) Vizzini collected from the natural environment-mixed forests of Southern Poland near Nowy Sącz-were used in the experiment. They were cleaned of plants and remnants and then lyophilized. The taxonomic identification was performed with MycoKey 4.1 software (http://www.mycokey.com) by an expert B. Muszyńska. Representative voucher specimens were deposited at the Department of Pharmaceutical Botany, Jagiellonian University Medical College, Krakow, Poland.

\section{Mycelial cultures}

To carry out the experiment, in vitro cultures of the abovementioned species were established on a modified liquid medium according to Oddoux [34].

The mycelial cultures were derived from the previously prepared explants of the fruiting bodies of A. bisporus and L. edodes of commercial origin as well as from $C$. cibarius and $I$. badia collected from the natural environment. For this purpose, a part of the hymenium was first defatted with $70 \%$ ethanol for several seconds, and the material was sterilized with a $15 \%$ solution of sodium chlorate for $2 \mathrm{~min}$. Then the fragments of fruiting bodies were rinsed several times with redistilled water and transferred to the Oddoux solid medium (laminar airflow). After 2 weeks of in vitro culture, $250 \mathrm{~mL}$ of the modified liquid medium (Oddoux) was transferred to the solid medium.

In the next stage of the experiment, mycelium from the in vitro cultures of L. edodes was transferred to $250 \mathrm{~mL}$ of the modified liquid medium containing $20 \mathrm{mg}$ of lovastatin in the form of a chemical compound (Sigma-Aldrich, Darmstadt, Germany), weighed on the analytical scales, and in the form of tablets of Liprox and Lovasterol preparations containing $20 \mathrm{mg}$ of lovastatin, respectively.

\section{Experimental cultures}

The cultures were established on the liquid medium to obtain the highest possible amount of biomass for use in further analyses. The cultures were shaken at $140 \mathrm{rpm}$ (Altel, Łódź) and then incubated at a temperature of $25 \pm 2{ }^{\circ} \mathrm{C}$ under a cycle resembling natural light conditions. Liquid in vitro cultures were shaken for 2 weeks, and then again transferred to a new medium to obtain a sufficient amount of biomass for the study.
After 2 weeks of establishing the in vitro culture of the tested mushroom species on the liquid medium, biomass was separated from the medium and rinsed several times with four-time distilled water. The obtained biomass was frozen and then lyophilized. The lyophilized samples were weighed.

\section{Preparation of artificial digestive juices}

A solution of artificial saliva with a pH of $6.7(4.355 \mathrm{~g}$ $\mathrm{K}_{2} \mathrm{HPO}_{4}, 3.407 \mathrm{~g} \mathrm{Na}_{2} \mathrm{HPO}_{4}, 15.018 \mathrm{~g} \mathrm{KHCO}_{3}, 5.844 \mathrm{~g}$ $\mathrm{NaCl}, 0.142 \mathrm{~g} \mathrm{MgCl}_{2}, 80.114 \mathrm{~g}$ citric acid, $1.665 \mathrm{~g} \mathrm{CaCl}_{2}$ ) [35] was prepared by dissolving the weighed contents in a small amount of four-time distilled water. Then the solution was transferred to a flask and topped up with water up to $1000 \mathrm{~mL}$.

A solution of artificial gastric juice with a $\mathrm{pH}$ of $2(2.0 \mathrm{~g}$ $\mathrm{NaCl}$ and $3.2 \mathrm{~g}$ pepsin) [36] was prepared by dissolving the weighed contents in a small amount of four-time distilled water. The solution was transferred to a flask, and then $80 \mathrm{~mL}$ of $1 \mathrm{M} \mathrm{HCl}$ was added. The solution was topped up with water up to $1000 \mathrm{~mL}$.

A solution of artificial intestinal juice with a $\mathrm{pH}$ of 8.0 $(0.020 \mathrm{~g}$ pancreatic extract, $0.125 \mathrm{~g}$ bile salts, and $8.4 \mathrm{~g}$ $\mathrm{NaHCO}_{3}$ ) [37] was prepared by dissolving the weighed contents in four-time distilled water and made up to $1000 \mathrm{~mL}$ with water.

\section{Digestion of lyophilized samples of fruiting bodies and biomass from in vitro cultures in artificial digestive juices}

An amount of $2.0 \mathrm{~g}$ of lyophilized fruiting bodies of $A$. bisporus, C. cibarius, I. badia, and L. edodes and their biomass was weighed. The contents were crushed and then transferred to flasks containing $3 \mathrm{~mL}$ of artificial saliva solution. The samples were incubated in this solution for $1 \mathrm{~min}$ (approximate physiological time of food residence in the oral cavity).

Then $20 \mathrm{~mL}$ of artificial gastric juice was added to each flask. The samples were extracted in this solution at $37{ }^{\circ} \mathrm{C}$ for $60 \mathrm{~min}$ in a Gastroel-2014 apparatus. The solutions were filtered through a tissue filter, and $20 \mathrm{~mL}$ of artificial intestinal juice was added to the biomass obtained. The solutions were incubated under similar conditions for $150 \mathrm{~min}$, and were filtered through tissue filters and then through membrane filters (Millex, Millipore Corporation, USA). The amount of lovastatin in the obtained filtrates was determined using the RP-HPLC method. 


\section{Digestion of $L$. edodes mycelium on lovastatin-enriched media in artificial digestive juices}

To carry out an experiment on media enriched with lovastatin, lyophilized biomass from in vitro cultures (enriched with lovastatin, Liprox, or Lovasterol) and the control sample were ground separately in a mortar. Then $2.0 \mathrm{~g}$ of the test portions was weighed and transferred to flasks. The contents of each flask were moistened with $3 \mathrm{~mL}$ of artificial saliva and left for $1 \mathrm{~min}$. Then the contents were decanted and $20 \mathrm{~mL}$ of gastric juice was added to each flask. In the next step, they were placed in the Gastroel-2014 apparatus, and the thermostat was set at $37^{\circ} \mathrm{C}$. Each sample was digested in the gastric juice for $60 \mathrm{~min}$. After an appropriate period of time, the contents of the flasks were filtered through pleated filter papers. The filtrate was left to determine lovastatin, and the digested materials together with the filter papers were placed in the flasks. Afterward, $20 \mathrm{~mL}$ of artificial intestinal juice was added for further digestion. The flasks containing the prepared samples were placed in the Gastroel-2014 device for $150 \mathrm{~min}$ each.

\section{Preparation of methanol extracts}

An amount of $2.0 \mathrm{~g}$ of lyophilized fruiting bodies of $A$. bisporus, C. cibarius, I. badia, and L. edodes and biomass from the in vitro cultures of these species was weighed. The contents were transferred to beakers containing $100 \mathrm{~mL}$ of methanol each. The samples were extracted using ultrasound (Polsonic Sonic 3, frequency $40 \mathrm{kHz}$ ) for $20 \mathrm{~min}$, and then the solutions were filtered into crystallizers. The obtained filtrate was evaporated and used for qualitative and quantitative determinations of lovastatin content using the RP-HPLC method.

\section{Determination of lovastatin content in mushroom material using the RP-HPLC method}

Chromatographic separation was performed using a highperformance liquid chromatography (HPLC) analyzer (Merck Hitachi). The process was carried out in an isocratic system with a mobile phase of constant composition. The apparatus was equipped with a UV detector $(\lambda=238 \mathrm{~nm})$, a column (Purospher RP18 14×200 mm, $5 \mu \mathrm{m}$ ), and a lamp (L7100). During each measurement, $20 \mu \mathrm{L}$ of the analyzed sample was injected, and the measurement was performed within $15 \mathrm{~min}$. All measurements were carried out using a previously prepared developing system [acetonitrile and $0.1 \%$ phosphoric acid in the ratio of $60: 40(\mathrm{v} / \mathrm{v})]$. The retention time of the standard substance was $12.75 \mathrm{~min}$.

Extracts obtained after the digestion of fruiting bodies and mycelium from the in vitro cultures of the tested mushroom species in artificial digestive juices were placed in crystallizers for evaporation. The dry residue was then dissolved in $1.5 \mathrm{~mL}$ of methanol and transferred to Eppendorf tubes using membrane filters (Millex, Millipore Corporation, USA). Extracts obtained after extraction using ultrasound and after evaporation were also dissolved in methanol, purified with membrane filters, and then analyzed.

The identification of the lovastatin was made by comparing the retention times of sample peaks with the standard compound. To confirm the presence of lovastatin in the tested extracts, the standard solutions of lovastatin was added as internal standards. The presence of the tested compound in the sample showed up as an increase in peak height for the appropriate retention time. The quantitative analysis of lovastatin was carried out using the calibration curve method. The chromatogram of lovastatin standard is presented in Fig. 2.

\section{Statistical analysis}

Statistical analyses were conducted using Microsoft Excel 2010 and the commercially available package GraphPad Prism v3.02 (GraphPad Software, San Diego, CA). The lovastatin concentrations in in vitro cultures of $L$. edodes were evaluated using one-way ANOVA test with Tukey's post hoc multiple comparisons. A $p$ value $<0.05$ was accepted as statistically significant. Data were presented as the mean \pm SD from three independent experiments $(n=3)$.

\section{Results and discussion}

\section{Determination of lovastatin content in the tested fruiting bodies and in vitro cultures}

The least squares linear regression was employed to statistically evaluate the changes in peak areas vs concentration of lovastatin. The calibration was made by the analysis of five standard solutions containing different concentrations of lovastatin in the range $0.0625-1 \mathrm{mg} / \mathrm{mL}$. The linear regression equation was $y=68756616 x+636261$. The determination coefficient $\left(R^{2}\right)$ obtained for the linear model was found to be 0.9997 . The LOD and LOQ values were $0.0144 \mathrm{mg} / \mathrm{mL}$ and $0.0435 \mathrm{mg} / \mathrm{mL}$, respectively.

The analysis of the obtained methanol extracts confirmed the presence of lovastatin in the tested species of edible mushrooms. The use of HPLC allowed determining the amount of lovastatin in the methanol extracts, which were obtained from the fruiting bodies of $A$. bisporus, $C$. cibarius, I. badia, and L. edodes as well as from the in vitro mycelial cultures of these species, and artificial digestive juices. After ultrasound extraction, lovastatin was determined both qualitatively and quantitatively in all the edible 


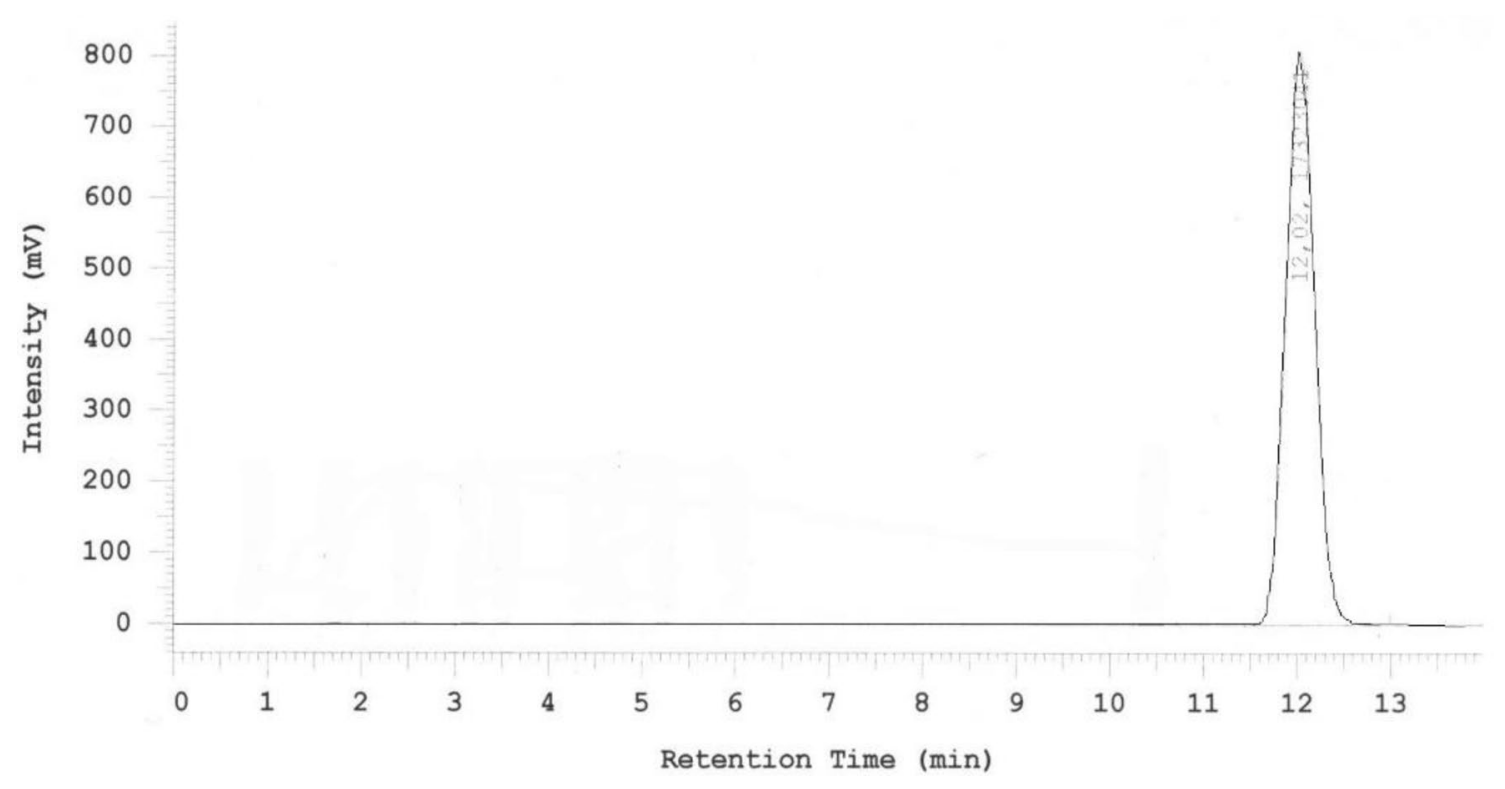

Fig. 2 Chromatogram of lovastatin standard

mushroom species investigated. The results of the analyses were expressed as the amount of lovastatin contained in $100 \mathrm{~g}$ of dry matter of each of the examined species. The amount of lovastatin in the methanol extracts obtained from the fruiting bodies of the studied species was estimated as follows: A. bisporus-30.79 mg/100 g d.w., $L$. edodes $-0.95 \mathrm{mg} / 100 \mathrm{~g}$ d.w., C. cibarius $-67.89 \mathrm{mg} / 100 \mathrm{~g}$ d.w., and I. badia-6.21 mg/100 g d.w. Similarly, the amount of lovastatin in the methanol extracts obtained from the in vitro mycelial cultures of the studied species was estimated as follows: A. bisporus $-47.96 \mathrm{mg} / 100 \mathrm{~g}$ d.w., $C$. cibarius-17.58 mg/100 g d.w., I. badia-16.57 mg/100 g d.w., and L. edodes-3.48 mg/100 g d.w. (Fig. 3).

The highest content of lovastatin was found in the fruiting bodies of $C$. cibarius species $(67.89 \mathrm{mg} / 100 \mathrm{~g} \mathrm{~d}$.w.) and the lowest in the fruiting bodies of L. edodes $(0.95 \mathrm{mg} / 100 \mathrm{~g}$ d.w.). An exemplary chromatogram for the determination of lovastatin in the methanol extract obtained from the fruiting bodies of $C$. cibarius is shown in Fig. 4.

A study by Chen showed that the fruiting bodies of many edible mushrooms contain significant amounts of lovastatin: A. bisporus $-56.54 \mathrm{mg} / 100 \mathrm{~g}$ d.w., Boletus
Fig. 3 Lovastatin content $(\mathrm{mg} / 100 \mathrm{~g} \mathrm{~d}$.w. $)$ in the lyophilized biomass of the mushroom species studied obtained after methanol extraction






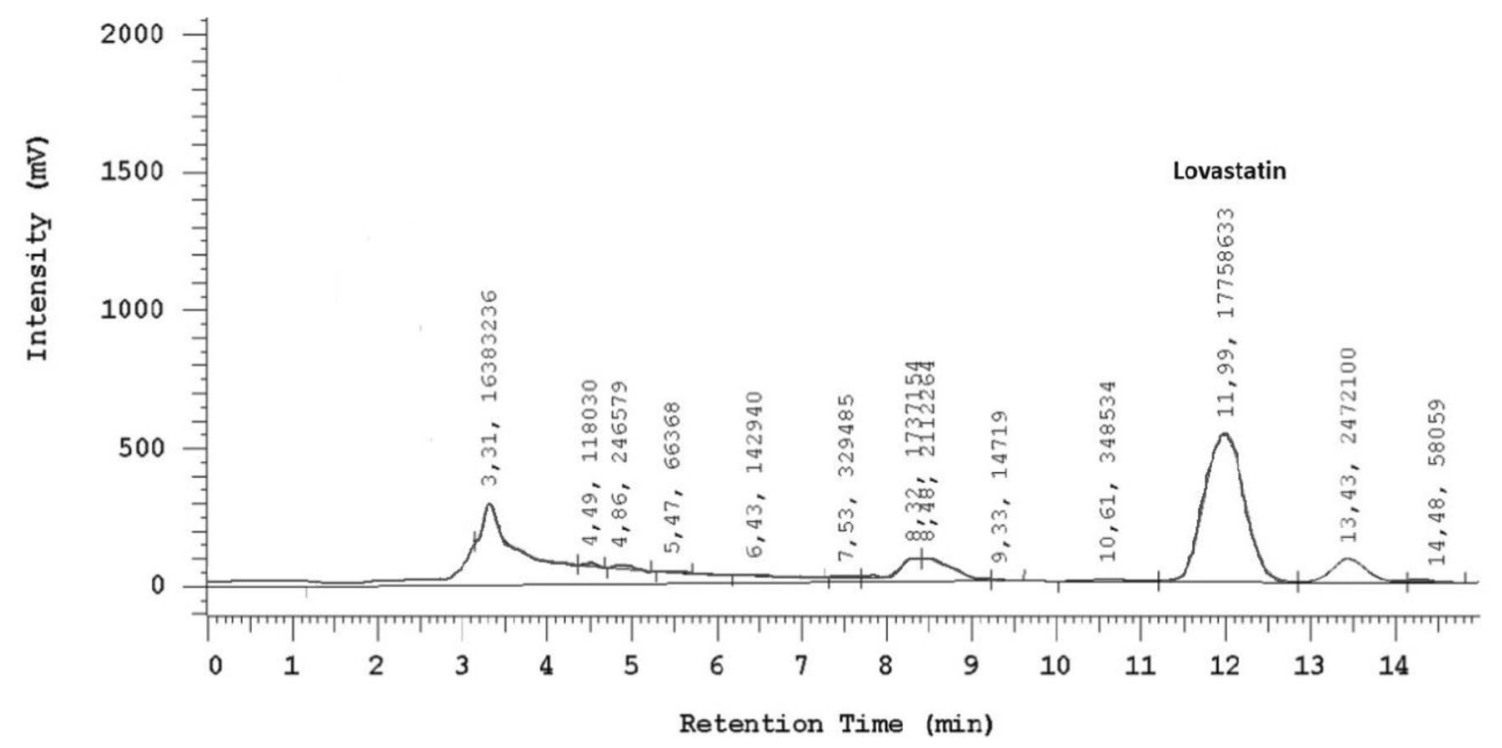

Fig. 4 Chromatogram obtained by analyzing a sample from the fruiting bodies of C. cibarius

edulis-32.73 mg/100 g d.w., and Pleurotus ostreatus-60.65 mg/100 g d.w [38]. The study also showed the amount of lovastatin in the fruiting bodies of edible mushrooms collected from different countries: Japan$16.53 \mathrm{mg} / 100 \mathrm{~g} \mathrm{d.w.,} \mathrm{Korea-21.64} \mathrm{mg/100} \mathrm{g} \mathrm{d.w.,} \mathrm{and}$ Taiwan-21.64 mg/100 g d.w. [38]. Lin et al. determined the amount of lovastatin in the fruiting bodies of $L$. edodes as $0.27 \mathrm{mg} / 100 \mathrm{~g} \mathrm{d.w.[30].} \mathrm{To} \mathrm{date,} \mathrm{P.} \mathrm{ostreatus}$ is most studied for lovastatin content because it is one of the edible mushroom species containing a significant amount of this compound. P. ostreatus exerts a hypocholesterolemic effect and was found to inhibit lipid peroxidation in rats and rabbits. It was demonstrated that a diet containing $10 \%$ of the dried fruiting bodies of $P$. ostreatus reduces the occurrence and size of atherosclerotic plaques [39]. To our knowledge, no study has determined the lovastatin content in the extracts from the fruiting bodies of C. cibarius and I. badia.

In the present study, a higher content of lovastatin was found in the extracts from in vitro mycelial cultures compared to the extracts from the fruiting bodies of mushroom species collected from the natural environment, except $C$. cibarius.

In another experiment, the content of lovastatin was determined in two types of in vitro cultures of $P$. ostreatus species (solid-state fermentation- $0.33 \mathrm{mg} / \mathrm{mL}$, in-depth fermentation- $0.74 \mathrm{mg} / \mathrm{mL}$ ) and their powdered fruiting bodies $(0.47 \mathrm{mg} / \mathrm{mL})$ [13]. Lin et al. determined the content of lovastatin in mycelium from the in vitro cultures of this species to be $2.22 \mathrm{mg} / 100 \mathrm{~g} \mathrm{~d}$.w.[30].

\section{Determination of lovastatin content after digestion of the test material in artificial digestive juice solutions}

The analysis of the obtained methanol extracts confirmed the presence of lovastatin in the tested mushroom species after extraction into artificial digestive juices. The results of the analysis were expressed as the amount of lovastatin released from $100 \mathrm{~g}$ of dry matter of each of the tested species. Both artificial gastric juice, after 60-min incubation preceded by incubation of the material in artificial saliva, and artificial intestinal juice, after 150-min incubation of the tested material preceded by incubation in artificial saliva and artificial gastric juice, were tested. An example of a chromatogram of lovastatin extracted from artificial digestive juices is shown in Fig. 5.

After incubation of the examined material in artificial gastric juice, lovastatin was determined qualitatively and quantitatively only for L. edodes $(0.02 \mathrm{mg} / 100 \mathrm{~g} \mathrm{d.w.}$. In the samples of the other species, the content of lovastatin was less than $0.01 \mathrm{mg} / 100 \mathrm{~g}$ d.w.

The release of lovastatin into artificial intestinal juice after 150 min of incubation (after a previous incubation in artificial saliva solution and artificial gastric juice) was confirmed in the samples of all the tested species of edible mushrooms, except the $A$. bisporus mycelium obtained from in vitro cultures; however, in most samples, the amount of lovastatin was below $0.01 \mathrm{mg} / 100 \mathrm{~g} \mathrm{~d} . \mathrm{w}$. The highest content of lovastatin was found in the extract from the in vitro cultures of L. edodes $(0.51 \mathrm{mg} / 100 \mathrm{~g} \mathrm{~d} . \mathrm{w}$.). Figure 6 shows 
Fig. 5 Example of a chromatogram obtained by the analysis of L. edodes extract from in vitro cultures on modified Oddoux medium after 60-min incubation in gastric juice and then 150 -min incubation in intestinal juice
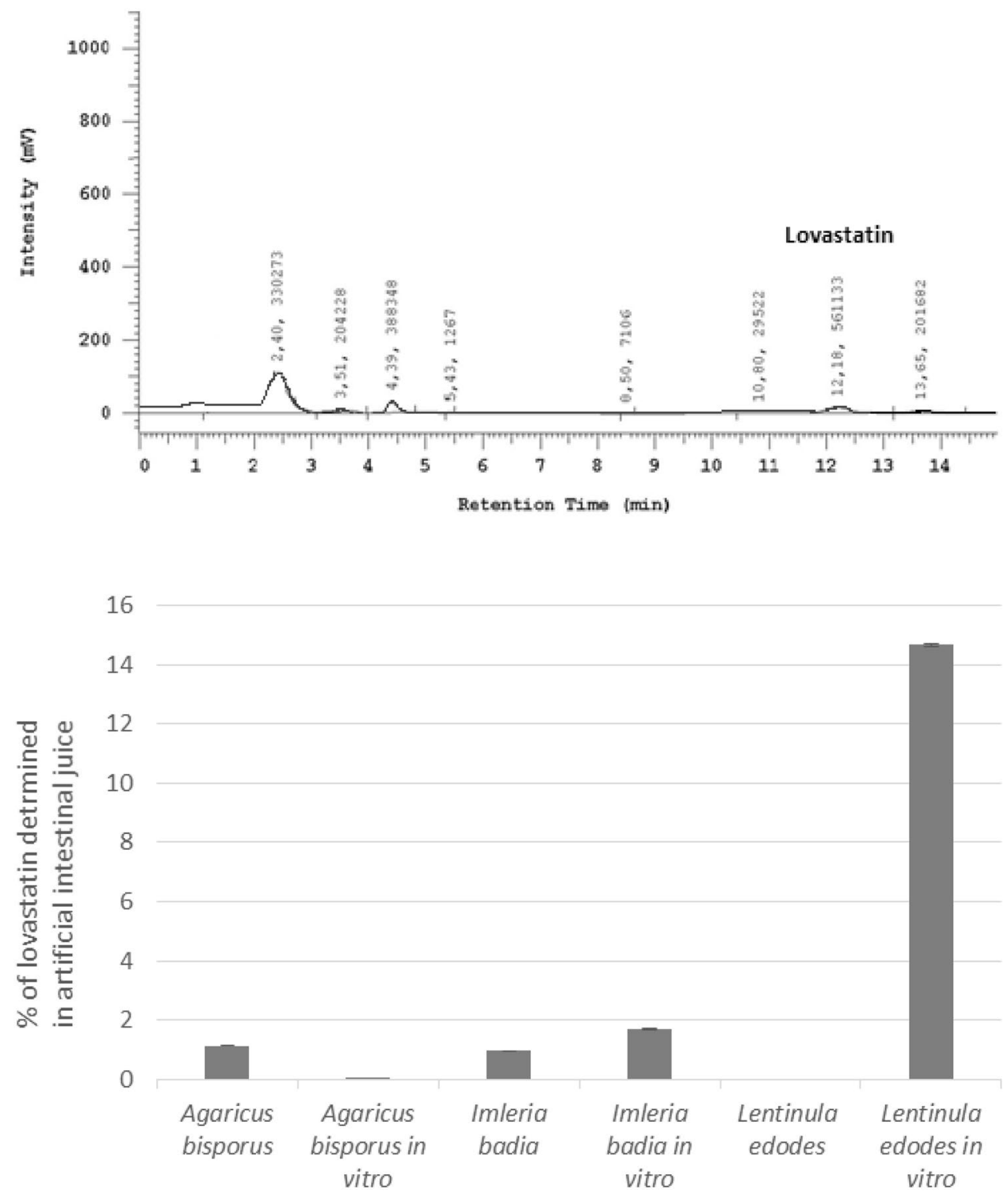

Fig. 6 Percentage of lovastatin determined in artificial intestinal juice in relation to its total content in the methanol extract of the mushroom materials tested the percentage of lovastatin determined in artificial intestinal juice in relation to the total content determined in the methanol extract of the mushroom materials studied. Because the in vitro cultures of $L$. edodes were found to have the highest content, this species was selected for further study.

\section{Evaluation of $L$. edodes ability to accumulate lovastatin}

The experiment showed that mycelium from the in vitro cultures of $L$. edodes established on a modified Oddoux medium enriched with lovastatin yielded a higher biomass than the control sample cultured without the addition of lovastatin. Biomass gains were as follows: $3.22 \mathrm{~g} / 250 \mathrm{~mL}$ medium for L. edodes + lovastatin, $1.65 \mathrm{~g} / 250 \mathrm{~mL}$ medium for $L$. edodes + Liprox, $1.62 \mathrm{~g} / 250 \mathrm{~mL}$ medium for $L$. edodes + Lovasterol, and $0.83 \mathrm{~g} / 250 \mathrm{~mL}$ medium for $L$. edodes (control sample). Although the highest amount of biomass was obtained from the in vitro cultures enriched with lovastatin, enrichment with commercial preparations also resulted in a significantly higher amount of biomass compared to the control sample. From the biotechnological point of view, this means that it is possible to obtain in vitro cultures of $L$. edodes with an increased hypocholesterolemic activity.

The analysis of the results showed that lovastatin is present in each of the studied species of edible mushrooms, but it is released into artificial digestive juices only in very small amounts. The highest content after incubation in artificial gastric juice was detected in the fruiting bodies of L. edodes $(0.02 \mathrm{mg} / 100 \mathrm{~g} \mathrm{~d} . \mathrm{w}$.) and after incubation in the intestinal juice in mycelium from the in vitro cultures of L. edodes (0.51 mg/100 g d.w.). 
To evaluate the ability of $L$. edodes mycelium to accumulate lovastatin, the content of this substance was determined in the mycelium from the in vitro cultures of this species established on Oddoux medium enriched with $20 \mathrm{mg}$ of lovastatin per $250 \mathrm{~mL}$ of medium or one tablet of Liprox or Lovasterol (20 mg of lovastatin) per $250 \mathrm{~mL}$ of medium. It was demonstrated that the in vitro cultures of $L$. edodes are capable of accumulating lovastatin. Its content in mycelium from the lovastatin-enriched medium was significantly higher compared to the control sample. The in vitro cultures on nonenriched medium contained $3.48 \mathrm{mg}$ of lovastatin per $100 \mathrm{~g}$ of dry matter, while those grown on enriched medium contained up to 100 times more lovastatin per $100 \mathrm{~g}$ of dry matter. Thus, significant differences $(p<0.05)$ were found in the content of lovastatin between the control L. edodes mycelium and the mycelium grown on Oddoux medium enriched with this substance or with one tablet of Liprox or Lovasterol. The most effective bioaccumulation of lovastatin was observed in the in vitro cultures enriched with pure substance compared to the two commercial preparations (Fig. 7).

\section{Examination of lovastatin release from mycelium from the in vitro cultures of $L$. edodes into artificial digestive juices}

It was shown that lovastatin from biomass obtained from the in vitro cultures of $L$. edodes on medium not enriched with the tested substance did not release into artificial digestive juices. Both in gastric and intestinal juice, lovastatin was found in amounts not exceeding the limit of quantification. In the case of in vitro cultures of $L$. edodes established on modified Oddoux medium enriched with lovastatin (both preparations and pure compound), lovastatin was found in intestinal juice after digestion of the tested material for $30 \mathrm{~min}$ in gastric juice. The results showed no clear correlation between the amount of lovastatin accumulated in the mycelium from in vitro cultures and the amount of lovastatin released into artificial digestive juices (Fig. 8). Studies on the bioavailability of biologically active substances from mushroom material are scarce. Several reports on the release of bioelements and other organic compounds from food including mushrooms into artificial digestive juices were published, but study of bioavailability of lovastatin from mushroom is a new research direction [40-43]. It is well known that lovastatin is present in edible mushrooms from the Basidiomycota taxon, and their regular consumption can lead to lowering cholesterol levels and thus preventing from cardiovascular diseases $[43,44]$. There are, however, reports on the bioavailability of lovastatin present in red yeast rice products [45]. It was proved that this natural material may contain lovastatin with a higher dissolution rate and reduced crystallinity compared to commercially available formulations, making it more bioavailable [45]. In addition, previous experiments on the bioavailability of lovastatin have shown that it is a substance with low bioavailability, which is why it is necessary to create forms of lovastatin that will allow the most effective absorption in the human body [45-47].

On the basis of the conducted analyses, it was found that the content of lovastatin was higher in the in vitro cultures than in the fruiting bodies of the tested mushrooms. This study proved the low availability of lovastatin after consumption, despite its high content in methanol extracts of the abovementioned species. Therefore, to utilize the antiatherosclerotic effect of mushrooms, alcoholic extracts are recommended.

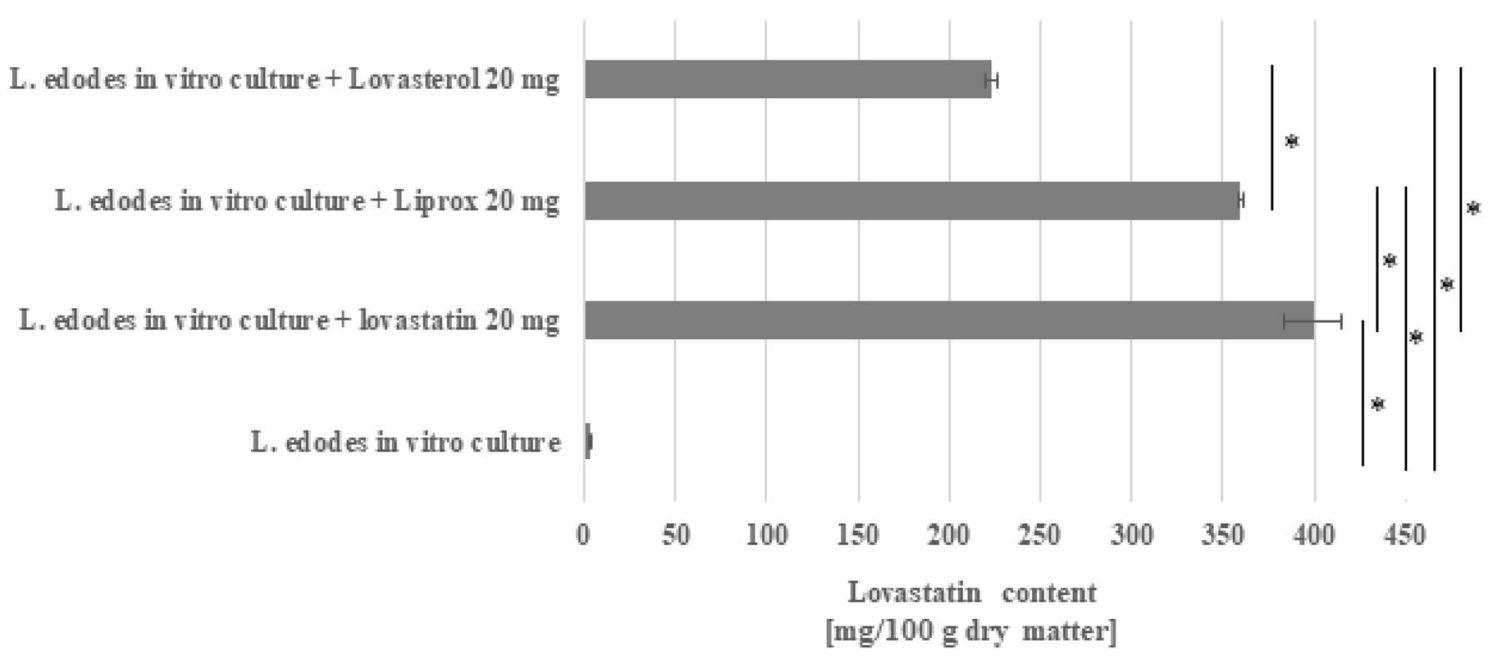

Fig. 7 Lovastatin content in biomass extracts from the in vitro cultures of $L$. edodes $(\mathrm{mg} / 100 \mathrm{~g}$ dry matter). The data are presented as a mean SD from three independent experiments $(n=3)$. For statistically significant results, $P<0.05$ 
Fig. 8 Lovastatin content extracted into artificial intestinal juice after digestion in gastric juice for 30 min from lyophilized biomass from the in vitro cultures of L. edodes (mg/100 g dry matter)

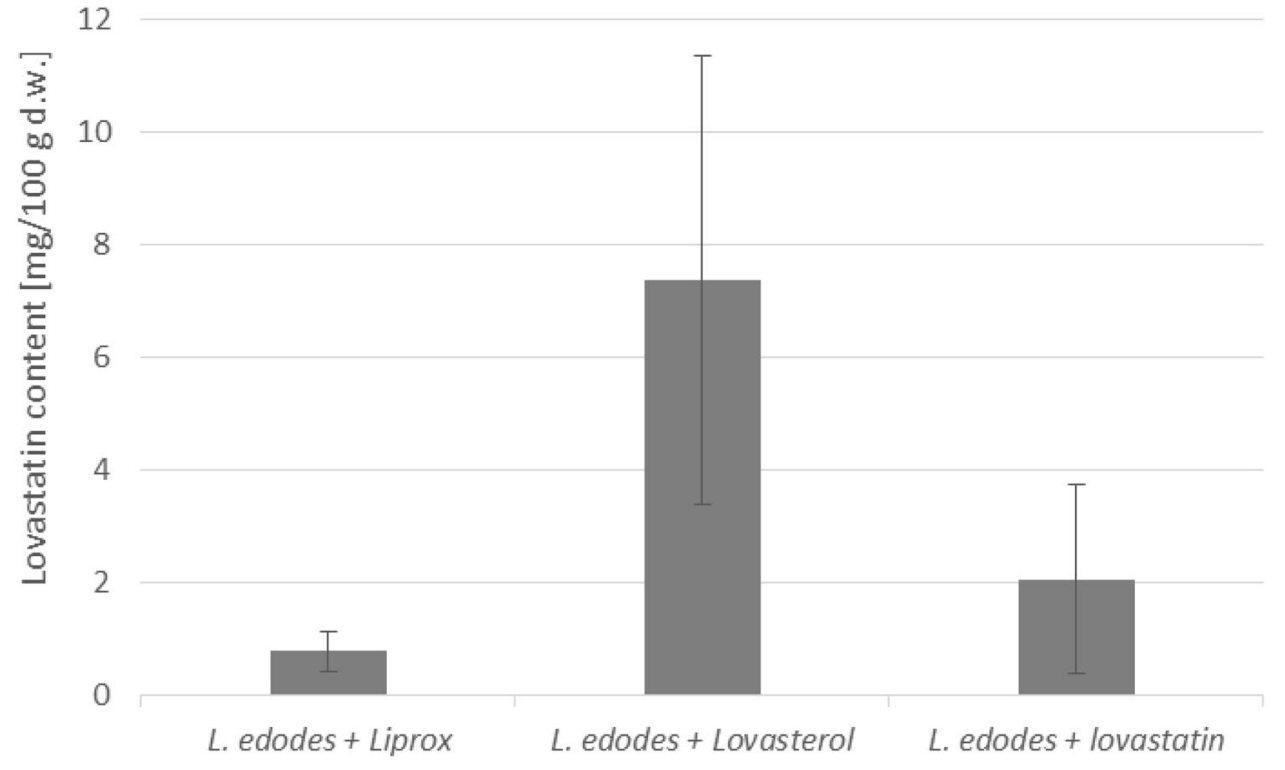

\section{Conclusion}

In vitro cultures of $L$. edodes effectively accumulated lovastatin and, therefore, the in vitro culture media can be supplemented with this compound to obtain mycelium with high hypocholesterolemic activity. Additionally, enrichment of the medium with lovastatin increased the biomass gains in relation to the cultures not enriched with this compound. The amount of lovastatin accumulated in mycelium from the medium enriched with commercial preparations was lower than that from the medium enriched with pure compound, but the ability to release the compound into artificial digestive juices differed between the cultures enriched with commercial preparations and pure substance.

\section{Compliance with ethical standards}

Conflict of interest On behalf of all authors, the corresponding author states that there is no conflict of interest.

Complance with ethics requirements This article does not contain any studies with human participants or animal performed by any of authors. No ethical approval was required.

Open Access This article is licensed under a Creative Commons Attribution 4.0 International License, which permits use, sharing, adaptation, distribution and reproduction in any medium or format, as long as you give appropriate credit to the original author(s) and the source, provide a link to the Creative Commons licence, and indicate if changes were made. The images or other third party material in this article are included in the article's Creative Commons licence, unless indicated otherwise in a credit line to the material. If material is not included in the article's Creative Commons licence and your intended use is not permitted by statutory regulation or exceeds the permitted use, you will need to obtain permission directly from the copyright holder. To view a copy of this licence, visit http://creativecommons.org/licenses/by/4.0/.

\section{References}

1. Kozarski M, Klaus A, Jakovljevic D, Todorovic N, Vunduk J, Petrović P, Niksic M, Vrvic MM, van Griensven L (2015) Antioxidants of edible mushrooms. Molecules 20:19489-19525

2. Muszyńska B, Grzywacz-Kisielewska A, Kała K, GdulaArgasińska J (2017) Anti-inflammatory properties of edible mushrooms: a review. Food Chem 243:373-381

3. Reshetnikov SV, Wasser SP, Tan KK (2001) Higher Basidiomycota as a source of antitumor and immunostimulating polysaccharides. Int J Med Mushrooms 3:361-394

4. Wasser SP (2002) Medicinal mushrooms as a source of antitumor and immunomodulating polysaccharides. Appl Microbiol Biotechnol 60:258-274

5. Elmastas M, Isildak O, Turkekul I, Temur N (2007) Determination of antioxidant activity and antioxidant compounds in wild edible mushrooms. J Food Compos Anal 20:337-345

6. Palacios I, Lozano M, Moro C, D’Arrigo M, Rostagno MA, Martínez JA, García-Lafuente A, Guillamón E, Villares A (2011) Antioxidant properties of phenolic compounds occurring in edible mushrooms. Food Chem 128:674-678

7. Sari M, Prange A, Lelley JI, Hambitzer R (2017) Screening of beta-glucan in commercially cultivated and wild growing mushrooms. Food Chem 216:45-51

8. Barros L, Cruz T, Baptista P, Estevinho LM, Ferreira ICFR (2008) Wild and commercial mushrooms as source of nutrients and nutraceuticals. Food Chem Toxicol 46:2742-2747

9. Kumar K (2015) Role of edible mushrooms as functional foods-a review. S Asian J Food Technol Environ 1:211-218

10. Muszyńska B, Piotrowska J, Krakowska A, Gruba A, Kała K, Sułkowska-Ziaja K, Kryczyk A, Opoka W (2017) Study of physiologically active components in different parts of fruiting bodies of varieties of Agaricus bisporus (white mushroom). Eur Food Res Technol 243:2135-2145 
11. Reis FS, Martins A, Vasconcelos MH, Morales P, Ferreira ICFR (2017) Functional foods based on extracts or compounds derived from mushrooms. Trends Food Sci Technol 66:48-62

12. Lee JW, Lee SM, Gwak KS, Lee JY, Choi IG (2006) Screening of edible mushrooms for the production of lovastatin and its HMG-CoA reductase inhibitory activity. Kor J Microbiol 42:83

13. Ramakrishnan M, Dubey C, Tulasi V, Kislai P, Manohar N (2017) Investigation of lovastatin, the anti-hypercholesterolemia drug molecule from three oyster mushroom species. Int J Biomed Clin Sci 2:26-31

14. Kaneda T, Tokuda S (1966) Effect of various mushroom preparations on cholesterol levels in rats. J Nutr 90:371-376

15. Kamiya T, Saito Y, Hashimoto M, Seki H (2002) Hypocholesterolemic alkaloids of Lentinus edodes (Berk.) Sing.- - a novel synthesis of eritadenine. Tetrahedron 28:899-906

16. Yang H, Hwang I, Kim S, Hong EJ, Jeung EB (2013) Lentinus edodes promotes fat removal in hypercholesterolemic mice. Exp Ther Med 6:1409-1413

17. Hajar R (2011) Statins: past and present. Heart Views $12: 121-127$

18. Walther U, Emmrich K, Ramer R, Mittag N, Hinz B (2016) Lovastatin lactone elicits human lung cancer cell apoptosis via a COX-2/ PPAR $\gamma$-dependent pathway. Oncotarget. 7:10345-10362

19. Oesterle A, Laufs U, Liao JK (2017) Pleiotropic effects of statins on the cardiovascular system. Circ Res 120:229-243

20. Wang Z, Li Y, Zhou F, Piao Z, Hao J (2016) Effects of statins on bone mineral density and fracture risk: a PRISMA-compliant systematic review and meta-analysis. Medicine (Baltimore) 95(22):e3042

21. Butterfield DA, Barone E, Mancuso C (2011) Cholesterol-independent neuroprotective and neurotoxic activities of statins: perspectives for statin use in Alzheimer disease and other age-related neurodegenerative disorders. Pharmacol Res 64:180-186

22. Das S, Mohanty M, Padhan P (2015) Outcome of rheumatoid arthritis following adjunct statin therapy. Indian J Pharmacol 47:605-609

23. Lim SY (2013) Role of statins in coronary artery disease. Chonnam Med J 49:1-6

24. Doumas M, Imprialos K, Dimakopoulou A, Stavropoulos K, Binas A, Athyros VG (2018) The role of statins in the management of nonalcoholic fatty liver disease. Curr Pharm Des. 24:4587-4592

25. Ziegler V, Albers A, Fritz G (2016) Lovastatin protects keratinocytes from DNA damage-related pro-apoptotic stress responses stimulated by anticancer therapeutics. Biochim Biophys Acta 1863:1082-1092

26. Matusewicz L, Meissner J, Toporkiewicz M, Sikorski AF (2015) The effect of statins on cancer cells-review. Tumour Biol 36:4889-4904

27. Bhargavi SD, Praveen VK, Marium S, Sreepriya M, Savitha J (2016) Purification of lovastatin from Aspergillus terreus (KM017963) and evaluation of its anticancer and antioxidant properties. Asian Pac J Cancer Prev 17:3797-3803

28. Zhao TT, Trinh D, Addison CL, Dimitroulakos J (2010) Lovastatin inhibits VEGFR and AKT activation: synergistic cytotoxicity in combination with VEGFR inhibitors. PLoS ONE 5:e12563

29. Wang D, Sun SQ, Wu WZ, Yang SL, Tan JM (2014) Characterization of water-soluble polysaccharide from Boletus edulis and its antitumor and immunomodulatory activities on renal cancer in mice. Carbohyd Polym 105:127-134

30. Lin SY, Chen YK, Yu HT, Barseghyan GS, Asatiani MD, Wasser SP, Mau JL (2013) Comparative study of contents of several bioactive components in fruiting bodies and mycelia of culinary-medicinal mushrooms. Int J Med Mushrooms 15:315-323

31. Kryczyk-Poprawa A, Żmudzki P, Maślanka A, Piotrowska J, Opoka W, Muszyńska B (2019) Mycoremediation of azole antifungal agents using in vitro cultures of Lentinula edodes. 3 Biotech 9:207

32. Kryczyk A, Piotrowska J, Sito M, Sulkowska-Ziaja K, Dobosz K, Opoka W, Muszyńska B (2017) Remediation capacity of Cd and $\mathrm{Pb}$ ions by mycelia of Imleria badia, Laetiporus sulphureus, and Agaricus bisporus in vitro cultures. J Environ Sci Health B 52:617-622

33. Muszyńska B, Dąbrowska M, Starek M, Żmudzki P, Lazur J, Pytko-Polończyk J, Opoka W (2019) Lentinula edodes mycelium as effective agent for piroxicam mycoremediation. Front Microbiol 10:313

34. Oddoux L (1957) Recherches sur les mycéliums secondairesdes Homobasidiés en culture pure. Imprimerie de Trevoux, Lyon, $\mathrm{p}$ 18

35. Arvidson K, Johasson EG (1985) Galvanic current between dental alloys in vitro. Scad J Dent Res. 93:467-472

36. Polish Pharmacopoeia (2014) Ed. X. PTFarm, Warszawa

37. Neumann M, Goderska K, Grajek K, Grajek W (2006) Modele przewodu pokarmowego in vitro do badań nad biodostępnością składników odżywczych. Żywność Technologia Nauka Jakość 1:30-45 (in Polish)

38. Chen SY, Ho KJ, Hsieh YJ, Wang LT, Mau JL (2012) Contents of lovastatin, $\gamma$-aminobutyric acid and ergothioneine in mushroom fruiting bodies and mycelia. LWT 47:274-278

39. Lindequist U, Niedermeyer THJ, Jülich WD (2005) The pharmacological potential of mushrooms. eCAM 2:285-299

40. Kafaoglu B, Fisher A, Hill S, Kara D (2016) Determination and evaluation of element bioaccessibility in some nuts and seeds by in-vitro gastro-intestinal method. J Food Compos Anal 45:58-65

41. Kała K, Krakowska A, Sułkowska-Ziaja K, Szewczyk A, Reczyński W, Opoka W, Muszyńska B (2017) Kinetics of extracted bioactive components from mushrooms in artificial digestive juices. Int J Food Prop 20:1796-1817

42. Muszyńska B, Kała K, Włodarczyk A, Krakowska A, Ostachowicz B, Gdula-Argasińska J, Suchocki P (2019) Lentinula edodes as a source of bioelements released into artificial digestive juices and potential anti-inflammatory material. Biol Trace Elem Res. https ://doi.org/10.1007/s12011-019-01782-8

43. Schneider I, Kressel G, Meyer A, Krings U, Berger RG, Hahn A (2011) Lipid lowering effects of oyster mushroom (Pleurotus ostreatus) in humans. J Funct Foods 3:17-24

44. Amirullah NA, Zainal Abidin N, Abdullah N (2018) The potential applications of mushrooms against some facets of atherosclerosis: a review. Food Res Int 105:517-536

45. Chen CH, Yang JC, Uang YS, Lin CJ (2013) Improved dissolution rate and oral bioavailability of lovastatin in red yeast rice products. Int J Pharm 444:18-24

46. Park K (2019) Shape-dependent bioavailability of lovastatin nanocrystals. J Control Release 307:423

47. Zhou J, Zhou D (2015) Improvement of oral bioavailability of lovastatin by using nanostructured lipid carriers. Drug Des Dev Ther 9:5269-5275

Publisher's Note Springer Nature remains neutral with regard to jurisdictional claims in published maps and institutional affiliations. 